\title{
Expectativas educativas y sociales de las familias inmigrantes
}

\author{
Jordi Garreta Bochaca
}

Universirat de Lleida. Facultat de Ciències de l'Educació

Complex La Caparrella. Partida La Caparrella. 25192 Lleida. Spain

\section{Resumen}

Analizada la importancia de las actitudes de los padres - expectacivas, niveles de aspiración, etc. - en el rendimiento escolar de los hijos, se presenta un estudio realizado entre famiIias procedentes de África residentes en Lérida. En éste se pondrán de manifiesto diferentes actitudes en función del proyecto familiar - producto de la trayectoria inmigrante- destacándose las observadas en el nivel de estudios deseado para los hijos, el nivel que realmente creen podrán alcanzar, la urilidad otorgada al sistema educativo y la movilización en la carrera discente.

Palabras clave: inmigración africana, educación, actitudes paternas de los inmigrantes, expectativas de los inmigrantes.

\section{Abstract. Social and education aspirations of immigrant families}

In this study we consider the analysis of the importance of parents attitudes - expectations and levels of aspiration- in the performance of children at school. This is a study carried out among families of African origin resident in Lérida. In this analysis different attitudes come out of the family study as a result of the immigrant phrnomenon. The following points stand out: the academic level desired for their children, the level that they really think can be achieved, the considered utility of the educational system and the importance of the pupil/teacher relationship.

Key words: african immigration, education, attitudes towards migrants, expectations towards migrants.

\section{Sumatio}

1. Introducción

2. Expectativas de los padres respecto a la escuela

3. Inmigración extranjera y educación
4. Conclusiones

5. Bibliografía 


\section{Introducción}

A partir de 1969, año en el que se publicó el Libro Blanco, el techo de las expectativas educativas de la familia española se ha ido elevando. Ya en el Informe FOESSA de 1970 , cerca de un 50\% de las amas de casa querían que sus hijos realizaran estudios superiores, aunque sólo el $26 \%$ esperaban ver realizado este deseo. Esta alta y creciente demanda educativa esta condicionada por dos percepciones sociales y una de individual (González Anleo, 1985): la primera de aquéllas corresponde a la rentabilidad de la educación como forma de mobilidad social; la segunda a la realidad evidente de una estructura socioeconómica diversa, diferenciada y desigual; por otro lado, González Anleo hace referencia a la aspiración individual que tiene como meta el triunfo social mediante la educación.

En trabajos postetiores (González Anleo, 1985; IEMB, 1992) se constata el incremento de las expectativas educativas, pero también puede observarse la diferencia existente entre deseos y realidad, siendo mayor esta diferencia en la clase media-baja y en la clase obtera.

De lo expuesto anteriormente podría parecer que estos trabajos han despertado el interés de los sociólogos o investigadores de otras disciplinas afines, esto no es así, el tema parece olvidado desde hace unos años. Coloma (1990) demandaba se le dedicara mayor atención, ya que sus resultados serían útiles para aclarar algunos aspectos de colaboración y conflicto en la relación familia/escuela.

El presente trabajo se inscribe dentro de esta línea, pero con tres particularidades:

1. La población - y en consecuencia la muestra de familias con hijos en edad escolar- queda delimitada a los inmigrantes africanos - $98,1 \%$ de los extranjeros (IOE 1992) - residentes en la provincia de Lérida.

2. Se complementa el primer objetivo vinculándolo al proyecto familiar -expectativas sociales-.

3. Utilización de una metodología cualitativa: entrevista en profundidad.

\section{Expectativas de los padres respecto a la escuela}

La familia esta considerada una de las agencias de socialización más importantes, dentro de este marco, la influencia familiar en el rendimiento escolar puede concretarse en los siguientes puntos (INCIE, 1976):

- la información que los padres tienen sobre los estudios y el comportamiento escolar de sus hijos;

- la actitud de los padres hacia la educación —expectativas, niveles de aspiración, interés por los estudios de sus hijos, etc.-一;

- la información y las actitudes paternales se reflejan en aspectos de la personalidad de los hijos que inhiben el trabajo como son la falta de compromiso personal con los estudios y la ausencia de motivación para estudiar. 
Los padres - y esto es especialmente relevante entre los inmigrantestransmiten sus experiencias y a partir de ellas proyectan sus expectativas e intentan modelar el futuro de sus hijos (Comas y Pujadas, 1991). Así pues uen la conformación de las demandas educativas, se puede constatar que existe un significativo grupo de alumnos "que sabe a qué atenerse", que tiene claro desde su niñez (EGB) que, de una u otra forma, va a llegar a la Universidad (lo cual no significa que sea un grupo motivado) porque ése es su "destino", un destino generalmente familiar y que se aferra a la enseñanza cualquiera que sea el resultado que obtenga. En el otro extremo se constata la presencia de un significativo grupo de alumnos que también "sabe a qué atenerse", y que ya desde su niñez "conoce" su destino y que éste no es llegar a la Universidad, sino a la "calle", que nada espera del, ni nada pide al, sistema de enseñanza. La relación que tienen estos grupos con la estratificación social de las familias es algo fuera de toda duda" (Fernández de Castro, 1990, p. 28).

En consecuencia, es obvia la importancia de las expectativas y actitudes de los padres en la conformación de la educación que recibirán los hijos. Estas expectativas paternas en cuanto a la función se pueden reducir en cuatro ámbitos (Oblinger, 1981; Coloma, 1990):

a) La escuela como lugar de aparcamiento de los hijos. En este ámbito estarían aquellos padres que cuentan que la escuela "guarde" a sus hijos durante un cierto número de horas los días laborables. San Roman nos facilita un ejemplo que también será útil para el segundo ámbito:

[...] a la pregunta sobre la utilidad de hecho de la escuela actual de sus hijos, el $69 \%$ responde que está en «aprender a leer y escribir", pero una quinta par confiesa que se soluciona el problema de que los niños anden por la calle o el problema de la comida, en las escuelas que tienen éste servicio. Un $7 \%$ opina que la escuela no sirve para nada a sus hijos y les impide que éstos ayuden en casa. (San Roman, 1986, p. 98-99).

b) La escuela como lugar de adquisición de conocimientos utilitarios. Este sería el primer nivel de expectativas de carácter pedagógico, referentes al objetivo instruccional. Los padres esperan que sus hijos reciban conocimientos útiles para su futura integración en el mundo del trabajo. Podemos constatar diferentes niveles en función de lo que se considere necesario por parte de los padres, existe un abanico de posibilidades, desde sólo el aprendizaje de lectura, escritura y matemáticas (ejemplo anterior), hasta las familias que amplían la definición a disciplinas más humanistas, como literatura, historia, arte, filosofia,etc.

c) La escuela como lugar de formación integral. En éste hallariamos las expectativas referentes al desarrollo de la persona en sus capacidades cognitivas y afectivas, desarrollo de la percepción y sensibilidad, logro del equilibrio interior y de la responsabilidad, afirmación de la autonomía personal y de la creatividad. Aquí se incluiría la transmisión de una u otra escala de valores. Este es el ámbito más diluido y conflictivo. 
d) La escuela como plataforma de movilidad social. Hallamos en éste las expectativas de los padres respecto al rendimiento escolar de los hijos como medio de ascender en la jerarquía social. Serían padres que acuden sólo a la escuela para protestar de las malas calificaciones de sus hijos sin interesarse por el aprendizaje. Este último ámbito puede darse conjuntamente con alguno de sus dos precedentes. Como se podrá constatar, la movilidad social es también finalidad implícita a toda petición de formación entre el colectivo de este estudio.

\section{Inmigración extranjera y educación}

Las expectativas y la actitud que tomen las familias condicionará, además de las diferencias sociales, económicas y culturales preexistentes, el éxito académico de las segundas generaciones de inmigrantes. Estas expectativas se inscriben dentro de lo que se ha llamado el proyecto migratorio (Zéroulou, 1988; Flitner, 1992).

A les élements potentiellement défavorables s'oppose, sur le plan de la motivation, l'intérêt marquué que les familles immigrés en général portent à la réussite scolaire de leurs enfants. Leurs ambirions dans ce domaine équivalent à celles de la moyenne allemande. [...]

Le grand intérêt pour la réussite scolaire de la seconde génerarion s'explique, selon Hopf (1990), par la sélectivité de la migtation; il s'agir, chez les migrants, d'une population relativement jeune, entreprenante et bien formée qui traduit, pour ainsi dire, les projects liés à leur prope migration en projects scolaires pour leurs enfants, cette traduction visant soit une continuation directe de leur prope histoire migratoire vécue comme réussie, soit, au contraire, une compensation quand les buts individuels de a migration n'ont pu être atteints (Flitner, 1992, p. 36-37).

Ante la ruptura del equilibrio familiat motivada por la inmigración, los padres adoptan diferentes conductas de adaptación que pueden incentivar o no el éxito escolar de sus hijos. Al emigrar, las familias llevan un proyecto y una voluntad de ascensión social, este proyecto inicial tiene como principal objetivo la acumulación de dinero para acceder a mejor posición social. Las vicisitudes que les depara la nueva situación pueden provocar que este proyecto de mejora se desplace en parte o totalmente hacia la escolarización de sus hijos esperando obtener mejores resultados. Estas familias consideran que los estudios de sus hijos son una inversión, como diría Bourdiett, adquisición de capital cultural que se transformará posteriormente en capital económico - este perfil responde a familias de procedencia urbana, mayor nivel cultural, menor tiempo de separación familiar (un año o dos como máximo antes del reagrupamiento), la idea de retorno no es acuciante, etc. (Zéroulou, 1988). Dentro de este grupo se sitúa parte de las familias inmigrantes entrevistadas en la provincia de Lérida, destacando la idea de no retorno y la movilización de los padres ante todo lo que sea educación de sus hijos. 
En las entrevistas realizadas ${ }^{1}$ se observa que las actitudes y expectativas se polarizan en función del proyecto familiar.

Un primer grupo (G1) serían aquellos individuos aún «ilusionados» en un proyecto, ya se trate del familiar -incluye a los hijos — o sólo aquellos que se centran en el de sus hijos, siendo el suyo no ya una mejora social, que desestiman, pero si una "supervivencia».

El segundo grupo de entrevistas (G2) lo conformarían los individuos con una actitud negativa, desilusionados -en todos los casos el cabeza de familia sufre inestabilidad laboral o se encuentra reiteradamente en el paro, deben recurrir a menudo a ayudas de instituciones no gubernamentales (Caritas Diocesana de Lérida, Creu Roja, etc.), piensan a menudo en el retorno-. En este grupo podemos corroborar la importancia otorgada a mantener al menos parte de la tradición del país de origen: «no quiere que hijos olviden la tradición musulmanan.

Entre estos dos grupos se observan algunas entrevistas que se sitúan en una transición (Gt) hacia la desilusión e idea de retorno, siempre en función de la situación laboral y económica del núcleo familiar. El límite de resistencia, hasta adoptar esta actitud, se situaría en aquel momento que necesitan ayuda - para sobrevivir, otras limitaciones económicas las constatan todos- de instituciones y no ven mejora en su situación.

En ninguno de los grupos se constatan diferencias en cuanto a profesión actual de los padres - - todos trabajadores sin cualificación-, tiempo transcurrido de separación familiar —entre la llegada del padre y el resto de la familia- y la edad de la pareja (todos entre 30 y 42 años) por el perfil del mismo colectivo en Lérida (Garreta, 1994).

\subsection{El nivel de estudios. Deseo/realidad}

El conocimiento sobre el sistema educativo español es bajo, pocos conocen más allá del curso que está realizando su/s hijo/s (en todos los casos EGB). No serfa necesario decir que la LOGSE (1990) es también una desconocida.

Previa explicación de las opciones, la diferencia se encuentra entre el nivel deseado y las posibilidades de conseguirlo:

- Los individuos del GI (entre los que se hallan todos los que han recibido algún tipo de formación en su país de origen), hablan de la universidad como meta —especialmente los estudios de Medicina - para sus hijos/as.

- Los integrantes del G2 y el Gt tienen menos aspiraciones, se inclinan por la Formación Profesional, estudios que consideran más útiles ante un hipotético regreso a su país de origen. La diferencia entre G2 y Gt radica en

1. El trabajo de campo ha consistido en entrevistas en profundidad, utilizando la estrategia de muestreo teórico, a familias inmigrantes — ambos cónyuges con hijos en edad escolar-. teriendo en cuenta el género, la procedencia, la residencia actual y la formación del entrevistado. 
que los primeros no se definen en el momento de responder si creen que podrán hacer realidad estos estudios - se remiten constantemente a "lo que les guste" a sus hijos-, en cambio en el Gt manifiesta "aún" el convencimiento de conseguirlo.

La actitud observada no difiere según el género del progenitor en ninguno de los grupos, a menudo el marido habla por boca de la entrevistada, las alusiones a éste son constantes. El género de los hijos resulta indiferente entre los individuos del primer grupo, mientras que en los otros dos se observa que la Formación Profesional demandada no es para las niñas que tienen otro futuro, el matrimonio.

\subsection{Utilidad del sistema educativo}

Los entrevistados se sitúan, en cuanto a sus expectativas, en dos de los ámbitos citados anteriormente. El primero y más importante es la percepción de la escuela como lugar de adquisición de conocimientos, presentándose una diferencia importante entre el G1, que amplía estos conocimientos hacia otras disciplinas más humanistas, y el G2 y Gt, que insisten en la importancia de la lengua, la escritura, las matemáticas y todo lo vinculado a la socialización ocupacional del estudiante, rechazando todo lo que distorsione la sustancia, la base de su tradición, que los padres intentan preservar en su casa.

Además de ser un lugar donde adquirir conocimientos, la escuela actuará como plataforma de movilidad social, la inversión económica que están realizando en sus hijos se convertirá en capital cultural que permite acercarse a una situación de igualdad, competir en el mercado laboral español y recuperar el capital invertido (GI); por otro lado, en G2 y Gt también se ve la escuela como plataforma de movilidad social, pero no en España sino en el pafs de origen, esta formación convertiría a sus hijos en privilegiados (Zeroulou, 1988).

\subsection{El impacto de la movilización familiar en la carrera discente}

La actitud de apoyo a la educación de los hijos esta extendida en el Gl y Gt, siendo menor esta percepción en el G2. Este apoyo se manifiesta principalmente en visitas a los profesores - dicen ir una vez al mes el GI y Gt y con menor frecuencia el G2-, colaboración en actividades extraescolares y ayuda en las tareas que realizan los niños en casa.

El interés de Gl va más allá, se plantean dar clases particulares a sus hijos, intención frecuentemente abortada por el precio de las mismas, esto lo resuelven en varios casos - no sólo en el GI sino también en el Gt- con la demanda al profesorado de especial atención para sus hijos. El profesorado no avala esta percepción, en definitiva el concepto de ayuda, visita a profesores, etc. es más restringido, ciñéndose a la solicitud del profesorado.

Los niños pueden constatar el interés de sus padres hacia sus estudios, la importancia que tiene este proyecto educativo en el seno de la familia, la pre- 
ocupación ante el fracaso escolar. La escuela se convierte en objeto de culto en el interior de la familia, es percibida como la institución que puede ayudarles a conocer $y$ comprender la sociedad en la que viven $y$, en consecuencia, aprender a moverse en ella. La voluntad de forzar su destino es una ambición que se concreta en un proceso de movilización material y moral favoreciendo la escolarización de los hijos (Zéroulou, 1988).

En el G2 la visita a la escuela se dilata en el tiempo, el interés por la educación de los hijos es menor, las clases particulares se consideran necesarias pero imposibles de costear - no se plantean alternativas-, el fracaso escolar es percibido de forma diferente - se acepta la repetición de curso sin solicitar al profesor pautas para ayudar al hijo-, etc., el problema mayor es la siruación económica familiar.

\section{Conclusiones}

La actitud —definida como expectativas, niveles de aspiración, interés por los estudios de los hijos, etc.- de los padres hacia la educación, es uno de los puntos donde se concentra la influencia familiar en el rendimiento escolar.

El proyecto familiar, producto de la trayectoria inmigrante condiciona esta actitud y, en consecuencia, las expectativas y la función otorgada a la escuela.

Esta primera aproximación al análisis de las actitudes y expectativas del colectivo inmigrante en la provincia de Lérida permite distinguir entre tres grupos:

- Grupo I (GI). Padres que "ilusionados" en el proyecto escolar de sus hijos, toman una actitud participativa y estimulante;

- Grupo 2 (G2). Padres udesilusionados» con la idea de retorno al país de origen, más aferrados a la tradición, poco motivadores y participativos;

- Grupo de transición (Gt). Situación intermedia que deriva haca el G2, pero con posibilidad de ser reversible.

La influencia de estas actitudes "positivas" y "negativas" no prescinde de la influencia de la variable origen social, éste es el principal de los obstáculos que deben superar, pero es necesario constatar que hay otros factores que determinarán el éxito o fracaso escolar de los hijos de inmigrantes extranjeros del llamado "Tercer Mundo", en este caso África.

\section{Bibliografía}

BRETON, J. y BELMONT, B. -CRESAS- (1985). Ouvertures; l'école, la creche, les familles. Parls: L'Harmatan.

COLECTIVo IOE (ACTIS, W.; PRADA, M.A. y PEREDA, C.) (1992). La inmigración extranjera en Cataluña. Barcelona: Institut d'Estudis Mediterranis.

- (1992). "La familia como agencia de socialización». En Sociologia de la educación. Madrid: Instituto de Estudios Económicos.

COlOMA, J. (1990). "La familia como agencia de socialización". En Fermoso, P. y otros. Sociología de la Educación, Barcelona: Alamex, p. 171-192. 
COMAS, D. y PUJADAS J.J. (1991). "Familias migrantes; reproducción de la identidad y del sentimiento de pertenencian. Papers, núm. 36, Barcelona: UAB.

COMISSIO D'ASSOCIACIONS I ORGANITZACIONS NO GOVERNAMENTALS DE LES COMARQUES DE GIRONA ( 9 de julio de 1992). Cinquanta propostes sobre immigració. Ciclostilado, Gerona.

ELEJABEITIA, C. (1991). "Los alumnos y sus padres ante la reforma de las enseñanzas medias". Educación y Sociedad, núm. 9, Madrid.

FEITO, R (1990). Nacidos para perder. Un análisis sociolbgico del rechazo y abandono escolares. Madrid: CIDE.

FERNÁNDEZ DE CASTRO, I. (1990). "Los procesos de enseñar y de aprender (algunas consideraciones sobre la desmotivación y el fracaso)". Educación y Sociedad, núm. 6, Madrid.

FLITNER-MERLE, E. "Les immigrés et l'école en Allemagne". Revue Française de Sociologie (enero-marzo, 1992). París: Editions du CNRS.

GARRETA, J. (1994). "Immigració extracomunitària i educació. La relació pares, escola i mestres". Revista llerda. Lérida: Institut d'Estudis Ilerdencs (adscrito CSIC) (en prensa).

GIROUX, H.A. y FLECHA, R. (1992), Igualdad educativa y diferencia cultural Barcelona: El Roure (colección Apertura).

GLASMAN, D. (1992). "Padres" o "familias", crítica a un vocabulario genèrico". Educación y Sociedad, núm. 11, Madrid.

GONZALEZ ANLEO, J. (1985). El sistema educativo Español. Madrid: Instituto de Estudios Económicos (colección Tablero).

IEMB (1992). Enquesta de la regió Metropolitana de Barcelona 1990, Condicions de vida i babits de la població. Vol. 4. Barcelona: Institut d'Estudis Metropolitans de Barcelona.

INCIE (Instituto Nacional de Ciencias de la Educación) (1976). Determinantes del rendimiento académico. Madrid: Ministerio de Educación y Ciencia.

LAPEYRONNIE, D. (1987). "Assimilation, mobilisation et action collective chez les jeunes de la seconde génération de l'immigration maghrébine». Revue Française de Sociologie (abril-junio, 1987). París: Editions du CNRS.

- (1992). Immigrés en Europe. Politiques locales d'integration. Patís: La documentation française.

LERENA, C. (1976). Escuela, ideologia y clases sociales en España. Barcelona: Editorial Ariel.

LYNCH, J. y PIMLOTT, J. (1979). Padres y profesores. Madrid: Anaya/2. (Colección Ciencias de la Educación).

OBLINGER, H. (1981). "Eltern und Familien als Einflussfaktoren». Die Schule in der Gesellschaft. Donawoerth: Veriag Ludwig Auer.

PAULSEN, R. (1991). "Education, social class, and participation in collective action" Sociology of Education. Vol. 64, núm. 2 (abril). A journal of the american sociological association.

PUJADAS, J.J. (1993). Etnicidad Identidad cultural de los pueblos. Madrid: Eudema.

SAN ROMAN, T. (1986). Entre la marginación y el racismo. Reflexiones sobre la vida de los gitanos. Madrid: Alianza Universidad (colección Ciencias Sociales).

TAPINOS, G.Ph. (1993). Inmigración e integración en Europa. Barcelona: Fundación Paulino Tortas Domenech.

ZEROULOU, Z. (1988), "La réussite scolaire des enfants d"immigrés". Revue Française de Sociologie (julio-septiembre, 1988). París: Editions du CNRS. 